\title{
A CRITICAL EXAMINATION OF THE COHERENCE-BASED GENEALOGICAL METHOD IN THE CATHOLIC EPISTLES ${ }^{1}$
}

\author{
Peter J. Gurry \\ (pgurry@ps.edu)
}

The present research provides the first sustained study of the Coherence-Based Genealogical Method (CBGM), a computerised tool developed by Gerd Mink which has become an 'essential tool' to the editors of the most widely used critical editions of the Greek New Testament (NA28/UBS5). Its main use has been on the Editio Critica Maior (ECM) for the Catholic Epistles, which now forms the basis of the NA and UBS editions. The ECM volume on Acts was published in 2017 and plans are underway to apply the CBGM to the entire New Testament. However, because it was designed to address the problems of textual contamination and coincidental agreement, the CBGM has significance far beyond the confines of biblical studies. The overarching purpose of the method is to improve our understanding of the text's history and to help reconstruct the text's starting point, or the 'initial text'. Both of these goals are subjected to close scrutiny in this thesis.

The argument unfolds in three sections. The first part traces the method's history and reception before offering a fresh, clear statement of its principles and procedures. Part II attends the method's claim to better reconstruct the initial text and Part III tests its ability to illuminate the text's historical development. A concluding chapter details limitations and suggests improvements. Here we offer a summary of each part.

The introduction sets out the aims and scope of the study. Chapter 1 then traces the development and reception of the method over the last thirty years, showing both that the method is mature enough to warrant scrutiny and in need of it. Chapter 2 clears the path for this by

1 A thesis submitted to the Faculty of Divinity, University of Cambridge, December 2016. 
clarifying the CBGM's goals, principles, and procedures. This fresh statement of the method is needed because the official explanations are often dense and cumbersome and because misunderstanding has ensued, as detailed in Chapter 1. This fresh expression of the method is especially written to be both accurate and clear. In the process, a number of examples are given which explain how the CBGM's use has led to textual changes in the new editions of the NA and UBS texts. A summary of changes between the NA27/UBS4, the first edition of the ECM, and the ECM2/NA28/UBS5 are given in this chapter. A full list of these changes is presented in Appendix A.

Chapters 3 and 4 begin Part II of the study and focus on the method's ability to reconstruct the Ausgangstext or 'initial text' as the proper goal of textual criticism. Chapter 3 addresses the current confusion about what the initial text is. We begin with a history of the term's use and then trace its subsequent confusion even among the editors of the ECM themselves. As a solution to the current debate, we argue that the term has been consistently used by its coiner, Gerd Mink, to mean 'the text from which our extant tradition originated', but that this definition allows it to refer to any number of historical entities including the author's original text, the text of the archetype, or something later such as an edited edition. As such, the term has value, but its proper referent needs to be delineated by each editor who uses it. The second section of Chapter 3 challenges the CBGM's claim to being a 'meta-method' by showing that the method is practically unnecessary for those who follow the Byzantine priority position even as it challenges key assumptions of thoroughgoing eclecticism. Instead, it is a tool designed by and best used by reasoned eclectics. In this section we also present the results of using the Byzantine text as the initial text in the CBGM. A final section of Chapter 3 explains why the use of pregenealogical coherence in the method to detect coincidental agreements is not viciously circular. This last point leads to Chapter 4, in which the CBGM is applied to the question of scribal habits in James. The results are compared with results from the same data, but utilising the widely used 'singular readings' method. The results show that the CBGM has distinct advantages over the use of singular readings. We argue that the use of singular readings to determine transcriptional probabilities should be abandoned in favour of a return to the use of practiced judgement, as in the work of Westcott and Hort. 
Chapters 5 and 6 shift to the more controversial claim of the CBGM to help us illuminate textual history. Chapter 5 begins by defending the CBGM's interest in such history against those who suggest that it is illsuited to or even uninterested in such history. To show otherwise, the rest of the chapter provides an extended historical test involving the text of the so-called Harklean Group of Greek witnesses and the Byzantine text. Despite the suggestion that the CBGM reverses their historical relationship, this study uses a newly produced portion of the global stemma to show otherwise. The results provide the first detailed example of how to use the CBGM to answer questions about historical relationships between texts. In doing so, we offer a lightly revised explanation for the origin of the Byzantine text in the Catholic Letters. Chapter 6 turns to a fundamental issue to all genealogical methods, namely, the question of what variants should be used to determine textual relationships. After considering several current proposals for determine genealogically significant variants, we discuss the specific cases of singular readings, orthographica, nonsense readings, and corrections. We argue that the CBGM's current practice is inconsistent in some of these cases and excludes valuable data in others. These cases, while not enough to invalidate the current version of the CBGM, should be revised in future versions.

The final chapter brings the thesis to a close by detailing three limitations before offering seven improvements. These limitations include the fact that contamination sometimes remains a problem for the CBGM, that history is not always parsimonious, and that the method can provide no shortcut to determine the cause (let alone a single cause) of variation. The improvements range from removing coincidental agreements to including more data, and improving the presentation of textual flow diagrams. Most important of all, we call for a public version of the method in which users can apply the CBGM to their own textual decisions rather than relying on the ECM editors' own decisions.

In the summary and conclusion of the study, we argue that the CBGM largely succeeds in its aims and that, with the revisions mentioned, it should continue to be used to edit the Greek New Testament and beyond. 